\title{
Hommage
}

\section{Camille Raichon 1942-2007}

\footnotetext{
I Institut national de la recherche agronomique (Inra) et les éditions Quae ont la tristesse de nous faire part du décès de Camille Raichon, ingénieur de recherche hors classe, survenu brutalement le 7 octobre 2007 dans sa $65^{\mathrm{e}}$ année.
}

\begin{abstract}
Jurassien d'origine, sociologue spécialisé dans le développement rural, Camille Raichon a réussi une carrière exemplaire au sein de l'Inra où il a débuté comme jeune scientifique en 1970. Spécialiste des systèmes de conduite d'élevages et de transformation des systèmes agraires, il a été rapidement reconnu et apprécié comme un professionnel éminent de la diffusion de la culture scientifique et technique. Dans cette voie il s'est intéressé à la création, à l'initiative de l'Agence universitaire de la Francophonie (AUF), d'une revue nouvelle "Les Cabiers d'études et de recherches francophones/Agricultures" dont le premier numéro est paru en mars-avril 1992. Il a participé longtemps aux activités du comité de rédaction jusqu'en 2006. Cette année-là, grâce à ses compétences, ses qualités humaines de médiateur, sa capacité de leader positif et généreux, il a été nommé Directeur exécutif des éditions Quae, une maison d'édition créée par l'Inra, l'Institut de recherche pour l'ingénierie de l'agriculture et de l'environnement (Cemagref), le Centre de coopération internationale en recherche agronomique pour le développement (Cirad) et l'Institut français de recherche pour l'exploitation de la mer (Ifremer).
\end{abstract}

Pour conduire à bien ce projet éditorial et managérial, Camille Raichon a toujours eu à cour de mener des partenariats diversifiés, équilibrés et toujours fondés sur la confiance et l'exigence de qualité. Camille Raichon a profondément marqué cette jeune structure par sa proximité complice et chaleureuse avec chaque agent participant à cette aventure.

À l'heure de se retirer il envisageait de reprendre une participation active au sein du comité de rédaction des Cabiers Agricultures. Nous évoquions cet avenir lors de notre dernière discussion, quelques jours avant sa disparition.

Camille Raichon laissera à tous le souvenir d'un dirigeant unanimement apprécié et d'un humaniste passionnément amoureux des livres et de leurs auteurs ainsi que celui d'un ami chaleureux que l'on avait toujours plaisir à rencontrer. 